\title{
Herramientas de Apoyo: El Portafolio (La experiencia de la Escuela de Medicina de Tec de Monterrey)
}

Francisco Ayala Aguirre, Graciela Medina Aguilar

Tecnológico de Monterrey, México

Al utilizar en educación superior estrategias de enseñanza - aprendizaje activas y participativas hace imprescindible el requerimiento de técnicas de evaluación que permitan medir la calidad, extensión y profundidad de los aprendizajes obtenidos por los alumnos expuestos a dichas estrategias.

El uso del portafolio en sus dos modalidades, electrónico e impreso, se ha vuelto un recurso valioso por la congruencia con modelos activos de educación centrados en el aprendizaje del alumno.

La experiencia de la Escuela de Medicina del Tecnológico de Monterrey al implantar un modelo educativo basado en competencias y un sistema de evaluación que incluye el portafolio como uno de sus recursos principales ha permitido corroborar estos planteamientos.

En este trabajo se describen las características, tipos y ventajas del uso del portafolio. Se presenta también

\footnotetext{
Correspondencia:

F. Ayala Aguirre

Departamento de Desarrollo Académico

Escuela de Medicina del Tecnológico de Monterrey

Campus Monterrey

Tel.: 52 (81) 88882031

fayala@itesm.mx

G. Medina: gmedina@itesm.mx
}

una experiencia específica del uso del portafolio en educación médica.

\section{INTRODUCCIÓN}

Los modelos educativos diseñados para el desarrollo de competencias llevan implícito el compromiso de la certificación del desarrollo de los conocimientos, habilidades y actitudes. Lo anterior es posible en la medida en que se logra tener evidencias tangibles de que se han logrado esos desarrollos. En este contexto, el portafolio como recurso de apoyo a un sistema de evaluación permite la identificación clara de las evidencias alineadas al desarrollo de las competencias, la reflexión sobre el desarrollo de cada competencia y por tanto, la posibilidad de la evaluación continua y de mejorar en el desempeño que están logrando los alumnos.

En este trabajo se integran las características, tipos y ventajas del uso del portafolio en educación superior, así como una experiencia de su implementación en el ámbito de la educación médica. 


\section{CARACTERÍSTICAS Y TIPOS DE PORTAFOLIO}

El Portafolio en el contexto de la educación superior, es un instrumento que tiene por objetivo demostrar la formación alcanzada en competencias, valiéndose de la selección fundamentada de las evidencias que debe reunir un alumno para lograr los objetivos de su formación en ciertas competencias cognitivas, procedimentales, actitudinales y de valores que serán necesarias para su práctica profesional.

En la mayor parte de las experiencias sobre el uso del portafolio se hace referencia a éste como parte del sistema de evaluación, es decir como una forma de documentación de la evidencias del alumno. Sin embargo, por lo común, se deja de lado la importancia de la reflexión sobre el proceso de documentación de las evidencias y su relación con el desarrollo de las competencias.

Las evidencias que se colectan en el portafolio, por una parte, son las que certifican que el alumno está logrando el aprendizaje y por tanto el desarrollo de las competencias. Por otra parte, permiten al docente el seguimiento del proceso de aprendizaje de los alumnos y por lo tanto también evaluar su propio proceso de enseñanza.

El sentido de aprendizaje del portafolio se encuentra en el proceso de justificación y reflexión que los alumnos hacen en torno a las evidencias que van colectando, en este proceso el alumno debe establecer la relación entre la evidencia, el aprendizaje logrado y el desarrollo de la competencia. En particular este proceso reflexivo en la conformación del portafolio permite que se le considere como una herramienta de evaluación continua y formativa ${ }^{1}$.

Una característica importante del uso del portafolio es que el alumno se hace responsable de su proceso de aprendizaje al asumir la tarea de la documentación de sus evidencias. En la medida en que debe decidir, reflexionar y documentar las evidencias relacionadas con el desarrollo de sus competencias podrá ir identificando sus niveles de avance e incluso trazarse un plan de mejora para lograr la competencia deseada.

Además de una forma de evaluación para el alumno ${ }^{2}$ la elaboración del portafolio le da la oportunidad al alumno de aprender a planificar y a autogestionar su aprendizaje a partir de las indicaciones del profesor. Le permite ser más autónomo en su proceso de aprendizaje, le motiva a ejercer la toma de decisiones durante la actividad de aprendizaje; el portafolio se convierte en una oportunidad de desarrollar la autorregulación de su proceso de aprendizaje.

Es posible identificar al menos cinco características que tipifican el modelo de portafolio, según Moya $^{3}$, que puede ser usado como una herramienta de tipo sistemático tanto en la planeación como en la evaluación del aprendizaje de los alumnos:

a. Englobador: Un abordaje global donde se usan técnicas de evaluación tanto formales como informales, enfocado tanto a procesos como productos de aprendizaje; se busca entender el desarrollo de habilidades, conocimientos, valores y aspectos afectivos en el alumno. Un portafolio que contiene aportaciones de carácter objetivo del alumno, del profesor y del tutor; que hace hincapié tanto en el desarrollo académico como en el desarrollo formativo del alumno.

b. Predeterminado y sistemático: implica el análisis profundo de las competencias profesionales de la carrera, de las evidencias que se deben lograr para el desarrollo de las competencias y del diseño de criterios o rúbricas de evaluación que permitan la evaluación de las evidencias. Todo lo anterior en el contexto de un diseño curricular integral que permita el logro gradual y lógico de las competencias que se desarrollarán en el alumno.

c. Informativo: La información contenida en las evidencias que se colectan en el portafolio debe tener significado para el alumno, para el profesor y para la institución educativa en general. Debe ser una fuente de información para actualización y mejoras al currículo.

d. Ajustable: Debe tener la posibilidad de ajustarse a condiciones de realidad, de acuerdo al propósito, metas y objetivos para el que es requerido.

e. Auténtico: Debe proporcionar información apegada a lo que realmente hace el alumno, es decir, debe mostrar lo que sucede en la realidad, reflejando lo que está aprendiendo a través de las prácticas y actividades que está realizando.

El portafolio resulta por sus características no solo la colección de información, implica la integración de experiencia y conocimiento por parte del alumno en un contexto curricular organizado $\mathrm{y}$ orientado a otorgar una formación integral y basada en el desarrollo de competencias para el ámbito profesional y para la vida.

Existen diferentes tipos de portafolios según sus 
características y usos, Shores y Grace ${ }^{4}$, proponen la siguiente clasificación:

Privado: es de carácter confidencial y requiere de lineamientos claros para su elaboración, sobre quienes serán sus usuarios y cómo se utilizará. Incluye reportes escritos elaborados principalmente por el docente y permiten tener una mayor comprensión de aspectos significativos con respecto a los trabajos incluidos en el portafolio de los alumnos.

De aprendizaje: es de carácter público y es el de uso más común, los alumnos definen con el docente lo que debe incluir. Contiene evidencias sobre el desarrollo de conocimientos, habilidades y actitudes. Hace posible la evaluación y apoyo al alumno en áreas específicas.

Continuo: es de carácter público pero restringido. Incluye la selección de una serie de trabajos clave del alumno por un período de tiempo específico. Hace posible el seguimiento en la formación del alumno por un período prologado de tiempo y por tanto la posibilidad de acciones de mejora.

Por otra parte, Danielason y Abrutyn ${ }^{5}$, proponen una tipificación de portafolios desde distinta perspectiva.

De trabajo: es una colección general y deliberada de evidencias de acuerdo a objetivos específicos. Es una forma de constatar las acciones realizadas por los alumnos.

De exhibición: contiene una selección de los mejores trabajos y lo que se busca es la demostración de los mayores avances realizados en un proceso de formación.

De evaluación diagnóstica: implica la documentación de aprendizajes del alumno y permite la retroalimentación sobre el desempeño del alumno. Facilita la toma de decisiones en base a qué tanto se han logrado los objetivos curriculares.

Aunque los tipos de portafolio se presentan como diferentes, es evidente que se pueden combinar y reorganizar según las necesidades de cada situación específica ${ }^{6}$.

\section{EL PORTAFOLIO EN EDUCACIÓN MÉDICA: LA EXPERIENCIA DE LA CARRERA DE MEDICINA DEL TEC DE MONTERREY}

La Escuela de Medicina del Tecnológico de Monterrey $^{\mathrm{a}}$, ha estado aplicando desde el año 2001 un modelo educativo basado en competencias, integrador de ciencias básicas y clínicas, que utiliza estrategias didácticas activas como el aprendizaje basado en problemas, aprendizaje colaborativo, aprendizaje orientado en proyectos y aprendizaje centrado en el paciente, con una exposición clínica temprana y con orientación a la comunidad.

Este modelo ${ }^{7}$ implica el desarrollo de competencias cognitivas, actitudinales, procedimentales y de valores, que en consecuencia requiere de su evaluación y seguimiento permanente de aprendizaje demostrable a través de evidencias específicas.

La Escuela ha recurrido al uso de portafolio (en sus versiones impresa y electrónica) como una herramienta que permite considerar holísticamente el proceso de enseñanza - aprendizaje que se esté desarrollando ${ }^{\mathrm{b}}$. La carrera se divide académicamente en tres departamentos: Ciencias básicas que comprende los tres primeros semestres; Ciencias Médicas Básicas que opera del cuarto al séptimo semestre; Ciencias Clínicas que organiza toda la formación clínica en los últimos dos años de la carrera bajo el esquema de trimestres y que en México se denomina internado rotatorio de pregrado.

La experiencia de evaluación utilizando el portafolio en las áreas de Ciencias Básicas y Médicas Básicas ha sido formativa más que sumativa. Al no tener valor sobre las calificaciones en ningún curso, ha permitido centrar los esfuerzos tanto de alumnos como de maestros en estimar el desarrollo de competencias de diversa índole, la extensión y profundidad del aprendizaje alcanzado, la calidad de metodología didáctica, la organización de las materias por Academias, así como los instrumentos de evaluación empleados.

$\mathrm{Al}$ cierre de cada uno de los primeros siete semestres, el alumno integra una carpeta de evidencias que demuestren el desarrollo de competencias cognitivas, manejo de procedimientos, la conducta ética y el desarrollo personal alcanzado por él.

Cada carpeta semestral debe contener un documento en el que el alumno describa su reflexión sobre los aprendizajes logrados, sus áreas deficientes y su plan de mejora.

Los maestros responsables de la revisión de estas carpetas enfocan su atención tanto en contenidos como en la forma en que están presentados,

\footnotetext{
a. http://dcs.mty.itesm.mx/

b. Estas decisiones se tomaron bajo las recomendaciones y asesoría del Comité de Evaluación, conformado por profesores de los distintos departamentos académicos de la Escuela de Medicina, con la coordinación y liderazgo de la Dra. Luz Leticia Elizondo.
} 
pero principalmente en la reflexión elaborada por el alumno y la coherencia que ésta tiene con las calificaciones obtenidas y la calidad de las evidencias presentadas.

Son ejemplos de piezas de evidencias solicitadas en los primeros siete semestres: Calificaciones obtenidas en diferentes materias; reporte del diagnóstico de salud de la comunidad en la que intervienen; protocolo de una investigación a desarrollar; documento (tipo publicación) de presentación de una investigación desarrollada; evaluaciones de sus tutores de las sesiones bajo el método didáctico de aprendizaje basado en problemas; reporte de dilemas éticos en que el alumno ha participado; evaluación utilizando listas de cotejo de procedimientos desempeñados en las guardias asignadas; entre otros.

Es importante resaltar que la oportunidad que el alumno tiene de recolectar piezas de evidencia y entregarlas a la administración académica para su revisión, sin un valor sumativo que se transforme en calificación, ha sido muy enriquecedora ya que estimula que el alumno se haga cargo de su propio proceso de aprendizaje.

Para los últimos dos años de la carrera, el departamento de Ciencias Clínicas organiza en cuatro áreas la formación de sus alumnos: Pediatría, Cirugía, Medicina Interna y Ginecología. La rotación por cada área dura 3 meses durante el primer año, para el segundo año los alumnos rotan en otras especialidades médicas.

En el 2001, Ciencias Clínicas implementó el uso de portafolio para los alumnos que estaban rotando en el área de Pediatría con el objetivo de realizar una evaluación multidimensional.

El alumno debía entregar las evidencias que demostraran el desarrollo de determinadas competencias clínicas así como su grado o nivel alcanzado. Entre otras evidencias debían contener las evaluaciones de los médicos responsables y residentes que hacían sobre el desempeño del alumno en sus cursos teóricos así como de las materias clínicas hospitalarias y ambulatorias.

Otro de los recursos de evaluación, además de los exámenes, era la entrega de los requerimientos solicitados en la herramienta llamada ALBALÁc, la cual consiste en documentar organizadamente las

c. Esta herramienta fue desarrollada por el Dr. Felicitos Leal, profesor de la Escuela de Medicina del Tec de Monterrey. experiencias de aprendizaje así como también la indagación de la información sobre dichas experiencias, utilizando para tal efecto el registro de historias clínicas, los procedimientos clínicos, la justificación teórica de esos procedimientos, la reflexión de los aprendizajes adquiridos y las áreas que el alumno debía reforzar.

La evaluación resultado del Portafolio, a diferencia de los primeros semestres de la carrera, determina la calificación en las materias Ambulatorias y Hospitalarias.

Una vez que se adquirió experiencia acerca de la aplicación de estos instrumentos y se definieron las políticas de evaluación con sus ponderaciones desglosadas, el Modelo se trasfirió a otros departamentos de tal manera que actualmente se aplica en la formación en Ciencias Clínicas en los dos años de Pregrado.

En sus inicios la inexperiencia y el alto nivel de exigencia del Comité de Evaluación de la Escuela, requería para la conformación del portafolio, una gran cantidad de productos en poco tiempo, generando en el alumno una fuerte ansiedad y afectando también al grupo de maestros tutores que debían revisarlos.

Aprendiendo de la implantación de este modelo, la escuela ha organizado de mejor modo la cantidad de las evidencias solicitadas, el tiempo invertido, los recursos docentes, las políticas de evaluación y las guías de instrucciones para los alumnos.

El uso de la herramienta ALBALÁ, antes descrita, fue en sus inicios con un formato electrónico. La falta de recursos propició que se dejara de utilizar la base de datos original. Actualmente se utiliza solo en formato impreso. La administración académica, conciente de la necesidad de volver al formato electrónico esta haciendo esfuerzos por utilizar la plataforma Blackboard que utiliza de manera institucional el Tecnológico de Monterrey para aprovechar mejor los recursos y ponerlos al servicio de la educación médica.

\section{CONCLUSIONES}

El aprendizaje que se logra al utilizar el portafolio puede considerarse como autodirigido ya que al conformarlo, los alumnos reflexionan sobre el aprendizaje que han obtenido, lo que en sí mismo modifica la percepción de la experiencia vivida. Este proceso permite integrar lo aprendido al conocimiento previo y le permite usarlo para iniciar un nuevo aprendiza- 
je. Todo este proceso es de particular riqueza en el contexto de la educación médica dónde el aprendizaje es un proceso activo y continuo.

El uso del portafolio en educación médica permite una evaluación basada en desempeño, lo cual da la oportunidad de dar seguimiento a los alumnos según van avanzando en el desarrollo de sus competencias.

Finalmente, se debe reconocer que en el proceso de planeación, diseño y revisión de los portafolios la institución educativa encuentra una excelente oportunidad de hacer una autoevaluación de la congruencia de su estructura curricular, de la claridad de las competencias que busca formar y de la calidad de las evidencias que puede exigir en la formación de sus alumnos.

\section{BIBLIOGRAFÍA}

1. Coll C, Martín E, Onrubia J. La evaluación escolar: dimensiones psicológicas, pedagógicas y sociales. Madrid: Alianza Editorial; 2004.

2. Barberà E, Bautista, G, Espasa A, Guasch T. Portafolio Electrónico: desarrollo de competencias profesionales en la red. Revista de Universidad y Sociedad del Conocimiento. 2006 Vol $3-\mathrm{N}^{\circ} 2$ Octubre. Disponible en: http://www.uoc.edu/rusc/

3. Moya Sh, O’Malley M. A Portafolio Assessment Model for ESL. The Journal of Educational Issues of Language Minority Students. 1994 Vol 13 p. 13-36, Spring.

4. Shores F, Grace C. The portfolio book, a step-by-step guide for teachers. Gryphon House, United States; 1998.

5. Danielson Ch, Abrutyn L. Una introducción al uso de portafolios en el aula. Fondo de Cultura Económica, México; 1999.

6. Webb C, Endacott R, Gray M, Jasper K, Miller C, McMullan M, Scholes J Models of portfolios. Medical Education. 2002 Vol 36,10: p. 897-898.

7. Wilkinson T., Challis M., Hobma S., Newble D., Parboosingh J., Sibbald R., The use of portfolios for assessment of the competence and performance of doctors in practice, Medical Education. 2002 Vol 36, 10: p. 918-924. 